\title{
Modelling heat transfer-controlled cooling and freezing times: a comparison between computational values and experimental results
}

\author{
A. La Rocca ${ }^{1}$, V. La Rocca ${ }^{2}$, A. Messineo ${ }^{3}$, M. Morale $^{2}$ \\ \& D. Panno ${ }^{2}$ \\ ${ }^{1}$ Department of Mechanical, Material and Manufacturing Engineering, \\ University of Nottingham, UK \\ ${ }^{2}$ Department of Energy, University of Palermo, Italy \\ ${ }^{3}$ Engineering and Architecture Faculty, University of Enna Kore, Italy
}

\begin{abstract}
Modelling of heat transfer-controlled cooling and freezing time predictions are very important for a good preservation of foodstuffs. In that regard, we used a computer code based on the finite-element method that allowed us to analyse the phase-change of various foodstuffs during their freezing. The model was exercised to predict process times. The results can be used to design high efficiency plants. In this work, the results predicted by the FEM program are compared with the experimental values given in technical literature.

Keywords: cooling, freezing, finite-element method, foodstuffs.
\end{abstract}

\section{Introduction}

The increment of world energy demand has brought an increment in greenhouse gas emissions with serious consequences on our environment [1, 2]. A reduction in greenhouse gas emissions can be achieved by increasing plant energy efficiency and by taking concrete actions on energy saving and recovery in the industrial field, e.g. in frozen food production and the storage sector.

Food conservation is one of the most common applications of freezing processes. Cooling and freezing of products reduces bacterial and microorganisms' activity, which-is responsible for food perishability. Production of high quality food is one of the major challenges for the frozen food industry. 
This industry is experiencing a significant increase in market demand; this induces companies to develop new processes. Design of appropriate freezing plants requires a knowledge of the cooling and freezing times of products [3-8]; these times can be determined through experimental measures or, more easily, through the development of analytical and numerical models.

Let $V$ be a finite volume element totally contained within the body of the considered food product and let $A$ be the surface enclosing this volume. Considering the Fourier equation, the conduction heat flow must comply with the internal energy variation of the volume per unit time:

$$
-\oint_{A} k \frac{\partial T}{\partial n_{A}} d A=\int_{V} C \frac{\partial T}{\partial t} d V
$$

where $k$ is the thermal conductivity, $C=\rho c$ is the specific heat capacity per unit volume and $\rho$ is the mass density of the material considered.

The finite-element method allows determining the unsteady temperature distribution. The volume $V$ is divided into a number of finite sub-volumes, $V_{1}$, $V_{2}, \ldots, V_{N}$, and the generic sub-volume $V_{i}$ must comply with the following:

- The temperature distribution and the physical properties (i.e.: thermal conductivity, $k$, specific heat capacity, $c$, and so on) will be determined by the local quantities $\left(T_{i}, k_{i}, c_{i}, \ldots\right)$ for an assigned point $P_{i}$ belonging to the sub-volume $V_{i}$.

- The boundary surface:

(a) belongs to the global volume boundary, $V$, and is denoted by $A_{i}$.

(b) represents the interface between the sub-volumes $V_{i}$ and $V_{j}$, and is denoted by $A_{i j}$.

- The segment connecting two points, $P_{i}$ and $P_{j}$, of the sub-volumes $V_{i}$ and $V_{j}$, respectively, have length $n_{i j}$ and it is normal to the surface $A_{i j}$. Let also denote by $m_{i j}$ the distance between $P_{i}$ and $A_{i j}$.

Let $F_{i}$ be the set of the j-indexes that satisfy the relationship $A_{i j} \neq 0$. Considering the thermal conductivity, $k=k(T)$, for each $j$ belonging to $F_{i}$ is:

$$
k_{i j}=k \frac{T_{i}\left(n_{i j}-m_{i j}\right)+T_{j} m_{i j}}{n_{i j}}
$$

In this way the more the surface $A_{i j}$ is far from $P_{i}$ the more temperatures are closer to $T_{j}$ rather than to $T_{i}$ and a linear behaviour can be assumed along the $P_{i} P_{j}$ segment. So equation (1) can be simplified and rewritten as:

$$
-\sum_{j \in F_{i}} k_{i j} \frac{T_{i}-T_{j}}{n_{i j}} A_{i j}=C_{i} V_{i} \frac{d T_{i}}{d t}
$$

satisfied for each $i=1,2, \ldots, M$ internal elements of the volume $V$.

For every $j$ of $F_{i}$ it can be written: $K_{i j}=\frac{k_{i j} A_{i j}}{n_{i j}}$ and equation (3) becomes: 


$$
T_{i}\left(-\sum_{j \in F_{i}} K_{i j}\right)+\sum_{j \in F_{i}} K_{i j} T_{j}=C_{i} V_{i} \dot{T}_{i}
$$

Setting $W_{i}=C_{i} V_{i}$ and expressing by $K_{i i}$ the part within the brackets in the previous equation, and, moreover, setting $K_{i j}=0$ when $j \notin F_{i}$, the system generated by equation (3) for $I=1,2, \ldots, M$ can be put in the matrix form:

$$
\left|\begin{array}{cccc}
K_{11} & K_{12} & \ldots & K_{1 M} \\
K_{21} & K_{22} & \ldots & K_{2 M} \\
\ldots & \ldots & \ldots & \ldots \\
K_{M 1} & K_{M 2} & \ldots & K_{M M}
\end{array}\right|\left|\begin{array}{c}
T_{1} \\
T_{2} \\
\ldots \\
T_{M}
\end{array}\right|=\left|\begin{array}{cccc}
W_{1} & 0 & \ldots & 0 \\
0 & W_{2} & \ldots & 0 \\
\ldots & \ldots & \ldots & \ldots \\
0 & 0 & \ldots & W_{M}
\end{array}\right|\left|\begin{array}{c}
\dot{T}_{1} \\
\dot{T}_{2} \\
\ldots \\
\dot{T}_{M}
\end{array}\right|
$$

or, more synthetically, in the compact form:

$$
K T=W \dot{T}
$$

Equation (6) does not completely describes the whole system, as it is not valid for the volume elements bounded by the surface $A i$, whose boundary conditions are not yet been defined.

\subsection{Boundary conditions}

Convection. Assuming the index $i$ as representative for an element of the boundary layer, and denoting by $h_{i}$ the convective coefficient and by $\vartheta_{i}$ the temperature of the fluid exchanging heat with the surface $A_{i}$, is:

$$
q_{i}{ }^{\prime}=h_{i} A_{i}\left(\vartheta_{i}-T_{i}\right)
$$

Moreover, considering the heat flows between $V$ and any of the confining elements $j \in \mathrm{F}_{\mathrm{i}}$, we have:

$$
h_{i} A_{i}\left(\vartheta_{i}-T_{i}\right)+T_{i}\left(-\sum_{j \in F_{i}} K_{i j}\right)+\sum_{j \in F_{i}} K_{i j} T_{j}=C_{i} V_{i} T_{i}
$$

Now, assuming $H_{i}=-h_{i} A_{i}$ and $B_{i}=h_{i} A_{i} \vartheta_{i}$, and denoting by $K_{i i}$ the sum within brackets in equation (8), the last equation becomes:

$$
T_{i}\left(K_{i i}+H_{i}\right)+\sum_{j \in F_{i}} K_{i j} T_{j}+B_{i}=C_{i} V_{i} T_{i}
$$

Equation (9) enables the application of equation (4) to the volume elements lying on the boundary layer with a convective heat exchange. In this case equation (4) can be considered as an isolated case of equation (9). Equation (9) can also be expressed in a matrix form, denoting by $H$ the diagonal matrix, $H_{i j}=H_{i} \delta_{i j}$, and by $B$ the $B_{i}$ : 


$$
(K+H) T+B=W T ;
$$

Heat flux. When the heat flux $q_{i}$ ” across the surface $A_{i}$ is known, we must compute it in the heat balance. This can be accomplished by means of a further generalization: in equation (9) we can put in this case: $B_{i}=\left(h_{i} \vartheta_{i}+q_{i}\right.$ ”) $A_{i}$.

Thermally insulated surface. This situation can be considered as an isolated case of the previous one where for $q_{i}$ " $=0$ and $h_{i}=0$ we have $B_{i}=0$.

Assigned nodal temperature. In some cases, in a given number of points (belonging to the surface or inside $V$ ) the time variation of temperature is known and/or fixed. In this case, we denote by $\mathrm{G}^{*}$ the set of the $j$ elements. So, for every $j$ belonging to $\mathrm{G}^{*}$ is $T_{j}=T_{j}^{*}=$ known constant.

Given the set I of the indexes $1,2, \ldots, N$, we can perform the thermal analysis of the assigned food product only for the elements for which the temperature is unknown. Hence, we neglect the $\mathrm{G}^{*}$-elements.

These are also necessary to take into account the heat transfer with the elements whose temperature has been assumed as known. For each $i \notin \mathrm{G}^{*}$, denote by $\mathrm{F}_{i}{ }^{*}$ the set $\mathrm{F}_{i} \cap \mathrm{G}^{*}$ (the condition $\mathrm{F}_{\mathrm{i}}{ }^{*}=\varnothing$ can also occur). Than $\mathrm{F}_{\mathrm{i}}{ }^{*}$ represents the set of element indexes confining with the element $i$ whose temperature is unknown. Equation (9) can be written:

$$
T_{i}\left(K_{i i}+H_{i}\right)+\sum_{j \in\left(F_{i}-F_{i} *\right)} K_{i j} T_{j}+\sum_{j \in F_{i} *} K_{i j} T_{j}^{*}+B_{i}=C_{i} V_{i} \dot{T}_{i}
$$

Equation (11) is valid for every $i \in\left(I-G^{*}\right)$, and equation (8) when $\mathrm{F}_{\mathrm{i}}^{*}=\varnothing$. Setting $D_{i}^{*}=\sum_{j \in F_{i}} K_{i j} T_{j}^{*}$ and introducing vector $\mathrm{D}^{*}=\left(D_{i}^{*}\right), i \in\left(\mathrm{I}-\mathrm{G}^{*}\right)$, we obtain the following matrix equation:

$$
(K+H) T+B+D^{*}=W \dot{T}_{i}
$$

which can be put in a more compact form by assuming $X=(K+H)$ and $E=(B$ $\left.+D^{*}\right)$ :

$$
X T+E=W \dot{T}_{i}
$$

\section{Numerical simulation of the freezing process and computational results}

The above described mathematical model was applied to predict the unsteady temperature field of some foodstuffs during the freezing process. For each of the considered foodstuffs the numerical results provided by the model were compared with experimental data found in the literature.

However, the lack of available data is a problem and they are often determined under particular conditions, therefore a comparison between analytical and experimental results is not an easy task. For this reason, we intend 
to perform experimental tests on several foodstuffs using new equipment located in our laboratories settled in the University of Palermo.

The reference experimental data have been obtained by several researchers by means of thermo-couple sensors located in a cylindrical sample (see Fig. 1) with different thickness $s$ and characterized by ratio thickness vs. diameter, $s / D<<1$, cooled by air streams that hit symmetrically the bases of the cylinder [9].

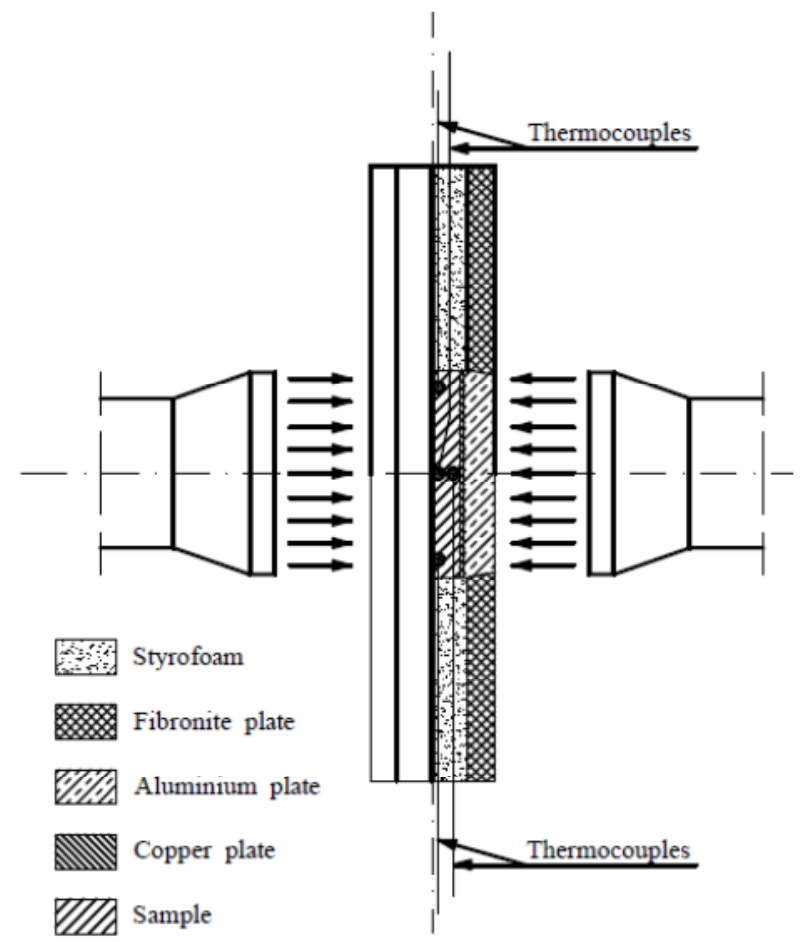

Figure 1: Cylindrical sample for experimental analysis.

A guard ring made up of an insulating material coated the lateral surface and a metallic plate located over the other two surfaces guaranteed an isothermal distribution of temperatures. This experimental arrangement makes the heat flow one-dimensional. Temperatures were measured mainly in two points:

- the centre of a base;

- the centre of the middle section.

By measuring the temperature at the external surface of the sample the convective coefficient was not needed; it was then possible to assume known temperature value as boundary condition. The main thermo physical data utilized in calculation are given in Tables 1 and 2.

Another problem concerning the foodstuffs data is related to variable calorimetric properties. This problem is due to the high content of water in some foodstuffs; this water content characterizes the freezing phenomena. To take into 
account this effect, as it has been proposed by several researchers [9-12], it is possible to fix a temperature interval $T_{L}$ and $T_{S}$, respectively the beginning crystallization temperature and the ending crystallization temperature, and then it is possible to assume a linear behaviour of thermal conductivity between them.

Table 1: $\quad$ Main parameters of analysed foodstuffs.

\begin{tabular}{|l|c|c|c|c|}
\hline Foodstuff & \multicolumn{2}{|c|}{ Specific heat capacity } & \multicolumn{2}{c|}{ Thermal conductivity } \\
\hline & \multicolumn{2}{|c|}{$[\mathrm{J} /(\mathrm{kg} \mathrm{K})]$} & \multicolumn{2}{c|}{$[\mathrm{W} /(\mathrm{m} \mathrm{K})]$} \\
\hline & $c_{L}$ & $c_{S}$ & $k_{L}$ & $k_{S}$ \\
\hline Mashed potatoes & 3517 & 1842 & 0.498 & 2.003 \\
\hline Shrimps & 3750 & 1951 & 0.534 & 2.069 \\
\hline Grouper & 3659 & 1942 & 0.531 & 2.048 \\
\hline Sole & 3659 & 1942 & 0.531 & 2.048 \\
\hline Cuttlefish & 3601 & 1925 & 0.524 & 2.004 \\
\hline Smooth hound & 3454 & 1883 & 0.508 & 1.895 \\
\hline
\end{tabular}

Table 2: $\quad$ Main parameters of analysed foodstuffs.

\begin{tabular}{|l|c|c|c|c|c|}
\hline Foodstuff & Density & \multicolumn{3}{|c|}{ Temperatures } & Thickness \\
\hline & {$\left[\mathrm{kg} / \mathrm{m}^{3}\right]$} & {$\left[{ }^{\circ} \mathrm{C}\right]$} & {$\left[{ }^{\circ} \mathrm{C}\right]$} & {$\left[{ }^{\circ} \mathrm{C}\right]$} & {$[\mathrm{m}]$} \\
\hline & $\rho$ & $T_{L}$ & $T_{M}$ & $T_{S}$ & $s$ \\
\hline Mashed potatoes & 1000 & -1 & -3 & -7 & 0.040 \\
\hline Shrimps & 1041 & -1 & -3 & -10 & 0.043 \\
\hline Grouper & 1036 & -1 & -3 & -12 & 0.045 \\
\hline Sole & 994 & -1 & -3 & -10 & 0.045 \\
\hline Cuttlefish & 965 & -1 & -3 & -10 & 0.045 \\
\hline Smooth hound & 1084 & -1 & -3 & -18 & 0.044 \\
\hline
\end{tabular}

The specific heat capacity in the given temperature interval has a peak, corresponding to the temperature $T_{M}$, and in this case it is possible to assume a linear behaviour among this three temperatures, known the peak value.

The assumed linear behaviour is in agreement with the experimental data. In this way, we can estimate all the needed variables with a small approximation.

\subsection{Model formulation}

The experimental setting show that the heat flux was can be assumed to be onedimensional. Denoting by " $x$ " the symmetry axis of the sample cylinder and setting the axis origin on the external surface, we can adopt for the temperature the following function: $T=T(x, t)$.

The input experimental data (boundary condition) in is $T_{0}^{*}(t)=T(0, t)$, so we have the evolution of the superficial temperature. This temperature is known at regular time intervals adequately spaced one from the other:

$$
T_{0} *(0), T_{0}^{*}\left(0+\Delta t_{1}\right), T_{0} *\left(0+\Delta t_{1}+\Delta t_{2}\right), \ldots, T_{0} *\left(0+\Delta t_{1}+\Delta t_{2}+\ldots+\Delta t_{N}\right)
$$

The time intervals $\Delta t_{n}(n=1,2, \ldots, N) . \Delta t_{n}$ is in the order of 250s and cause insufficient accuracy and unstable solutions and cannot be used directly. A time 
interval, $\Delta t$, small and sub multiple of the $\Delta t_{n}$ was defined; a time interval of 5 or 10 seconds was chosen.

The evolution of $T_{0}^{*}$ during each $\Delta t_{n}$ have to be evaluated. A linear behavior between the extremes of each time interval was inferred.

Denoting by $m$ an integer that realizes the condition $m \Delta t \leq \Delta t_{n}$, in a generic instant belonging to the $n$-th time interval, it results:

$$
\begin{aligned}
& T_{0} *\left(0+\Delta t_{1}+\Delta t_{2}+\ldots+\Delta t_{(n-1)}+m \Delta t\right)=T_{0} *\left(0+\Delta t_{1}+\Delta t_{2}+\ldots+\Delta t_{(\mathrm{n}-1)}\right)+ \\
& +\left[T_{0} *\left(0+\Delta t_{1}+\Delta t_{2}+\ldots+\Delta t_{n}\right)-T_{0} *\left(0+\Delta t_{1}+\Delta t_{2}+\ldots+\Delta t_{(n-1)}\right)\right] m \Delta t / \Delta t_{n}(15
\end{aligned}
$$

Setting now $M_{n}=\frac{\Delta t_{n}}{\Delta t}$ (for $\left.n=1,2, \ldots ., N\right)$, equation (15) give the value of $T_{0} *\left(\left[0+M_{1}+M_{2}+\ldots+M_{(n-1)}+m\right] \Delta t\right)$ which can be used to set the boundary condition at the $\left(M_{1}+M_{2}+\ldots+M_{(n-1)}+m\right)$-th iteration. In this way the temperatures $T_{0}^{[i]} *=T_{0} *(i \Delta t)$ is fixed.

The cylindrical sample is divided into 11 elements. Taken into consideration the physical and boundary symmetry, only 6 elements and their nodes for the temperature computation were considered:

$$
x_{0}=0 ; x_{1}=\frac{s}{10} ; x_{2}=\frac{2 s}{10} ; \ldots \ldots \ldots \ldots ; x_{5}=\frac{5 s}{10}=\frac{s}{2}
$$

The distance between two adjacent nodes is $\Delta x=s / 10$. Each element is defined by the lateral surface of a cylinder having unitary base surface and axis the $x$ direction, and by the planes having equations:

$$
x=0 ; x=\frac{\Delta x}{2} ; x=3 \frac{\Delta x}{2} ; x=5 \frac{\Delta x}{2} ; \ldots \ldots ; x=11 \frac{\Delta x}{2}
$$

The conduction heat transfer from one element to the other is across these planes. Retrieving the FEM notation we have:

$$
\mathrm{I}=\{0,1,2,3,4,5\} ; \mathrm{G}^{*}=\{0\} ; \mathrm{I}-\mathrm{G}^{*}=\{1,2,3,4,5\} ; \mathrm{p}=5
$$

The superficial element " 0 ", whose temperature is known for each instant, (0 $\in \mathrm{G}^{*}$ ), is the bound for all the other elements. So, using the usual notation, it can be written:

$$
\begin{aligned}
& A_{01}=A_{12}=A_{23}=A_{34}=A_{45}=1 \\
& n_{01}=n_{12}=n_{23}=n_{34}=n_{45}=\Delta x
\end{aligned}
$$

Remembering now that $k_{i j}$ denotes the temperature value on the common surface $A_{i j}$ pertaining to $i$ and $j$ nodes, it follows

$$
k_{i j}^{[s]}=k\left(T_{i j}^{[s]}\right) \approx k\left(\left[\left(\mathrm{~T}_{\mathrm{i}}(s \Delta t)+T_{j}(s \Delta t)\right] / 2\right)\right.
$$

Supposing a linear behaviour of temperatures between the nodes. Thermal conductivity is a time function too. So it is:

$$
c^{[s]}{ }_{i}=c\left(T_{i}(s \Delta t)\right)
$$

The fundamental equation for the particular case studied is: 


$$
\begin{aligned}
& \frac{\Delta t}{\Delta x}\left[\begin{array}{ccccc}
-\left(k_{01}^{[i]}+k_{12}^{[i]}\right) & k_{12}^{[i]} & 0 & 0 & 0 \\
k_{12}^{[i]} & -\left(k_{12}^{[i]}+k_{23}^{[i]}\right) & k_{23}^{[i]} & 0 & 0 \\
0 & k_{23}^{[i]} & -\left(k_{23}^{[i]}+k_{34}^{[i]}\right) & k_{34}^{[i]} & 0 \\
0 & 0 & k_{34}^{[i]} & -\left(k_{34}^{[i]}+k_{45}^{[i]}\right) & k_{45}^{[i]} \\
0 & 0 & 0 & 2 k_{45}^{[i]} & -\left(2 k_{45}^{[i]}\right)
\end{array}\right] \times\left[\begin{array}{c}
T_{1}^{[i]} \\
T_{2}^{[i]} \\
T_{3}^{[i]} \\
T_{4}^{[i]} \\
T_{5}^{[i]}
\end{array}\right]+ \\
& +\frac{\Delta t}{\Delta x}\left[\begin{array}{c}
k_{01}^{[i]} T_{0}^{*[i]} \\
0 \\
0 \\
0 \\
0
\end{array}\right]=\rho \Delta x\left[\begin{array}{ccccc}
c_{1}^{[i]} & 0 & 0 & 0 & 0 \\
0 & c_{2}^{[i]} & 0 & 0 & 0 \\
0 & 0 & c_{3}^{[i]} & 0 & 0 \\
0 & 0 & 0 & c_{4}^{[i]} & 0 \\
0 & 0 & 0 & 0 & c_{5}^{[i]}
\end{array}\right]\left(\left[\begin{array}{c}
T_{1}^{[i+1]} \\
T_{2}^{[i+1]} \\
T_{3}^{[i+1]} \\
T_{4}^{[i+1]} \\
T_{5}^{[i+1]}
\end{array}\right]-\left[\begin{array}{c}
T_{1}^{[i]} \\
T_{2}^{[i]} \\
T_{3}^{[i]} \\
T_{4}^{[i]} \\
T_{5}^{[i]}
\end{array}\right]\right)
\end{aligned}
$$

the previous equation produces the following results for this isolated case:

$$
\begin{aligned}
& T_{1}^{[\mathrm{i}+1]}=T_{1}{ }^{[\mathrm{i}]}+\left[\Delta t /\left(\rho c_{1}{ }^{[\mathrm{i}]} \Delta x^{2}\right)\right]\left[k^{[\mathrm{ij}]}{ }_{01}\left(T_{0}{ }^{[\mathrm{i}]}-T_{1}^{[\mathrm{i}]}\right)+k^{[\mathrm{i}]}{ }_{12}\left(T_{2}{ }^{[\mathrm{i}]}-T_{1}{ }^{[\mathrm{i}]}\right)\right] \text {; } \\
& T_{2}{ }^{[\mathrm{i}+1]}=T_{2}{ }^{[\mathrm{i}]}+\left[\Delta t /\left(\rho c_{2}{ }^{[\mathrm{i}]} \Delta x^{2}\right)\right]\left[k^{[\mathrm{i}]}{ }_{12}\left(T_{1}{ }^{[\mathrm{i}]}-T_{2}{ }^{[\mathrm{i}]}\right)+k^{[\mathrm{i}]}{ }_{23}\left(T_{3}{ }^{[\mathrm{i}]}-T_{2}{ }^{[\mathrm{i}]}\right)\right] \text {; } \\
& T_{3}{ }^{[\mathrm{i}+1]}=T_{3}{ }^{[\mathrm{i}]}+\left[\Delta t /\left(\rho c_{3}{ }^{[\mathrm{i}]} \Delta x^{2}\right)\right]\left[k^{[\mathrm{i}]}{ }_{23}\left(T_{2}{ }^{[\mathrm{i}]}-T_{3}{ }^{[\mathrm{i}]}\right)+k^{[\mathrm{i}]}{ }_{34}\left(T_{4}{ }^{[\mathrm{i}]}-T_{3}{ }^{[\mathrm{i}]}\right)\right] \text {; } \\
& T_{4}{ }^{[i+1]}=T_{4}{ }^{[i]}+\left[\Delta t /\left(\rho c_{4}{ }^{[i]} \Delta x^{2}\right)\right]\left[k^{[i]}{ }_{34}\left(T_{3}{ }^{[i]}-T_{4}{ }^{[i]}\right)+k^{[i]}{ }_{45}\left(T_{5}{ }^{[i]}-T_{4}{ }^{[i]}\right)\right] \text {; } \\
& T_{5}{ }^{[\mathrm{i}+1]}=T_{5}{ }^{[\mathrm{i}]}+\left[\Delta t /\left(\rho c_{5}{ }^{[\mathrm{i}]} \Delta x^{2}\right)\right]\left[2 k^{[\mathrm{i}]}{ }_{45}\left(T_{4}{ }^{[\mathrm{i}]}-T_{5}{ }^{[\mathrm{i}]}\right)\right] \text {; }
\end{aligned}
$$

Subsequent iterations enable prediction of the temperature profile at the centre of the sample. The theoretical and experimental temperature for the instants $i=M 1, M 1+M 2, \ldots$. , can now be compared. Finally the theoretical and experimental time-temperature curves for the freezing of several foods are given in Figure 2.

A good agreement between the theoretical and experimental curves can be seen.

The density variation in the foodstuffs during the crystallization was not addressed in the present model; this together with the approximation of the thermophysical properties of the foodstuffs during the phase change has contributed to the deviations between experimental and predicted values.

\section{Conclusions}

The present work represents a first step as part of a bigger project. Indeed, the numerical scheme proposed by the authors has been validated using experimental data found by several references; nevertheless, the proposed model is in good agreements with such data. The proposed numerical approach is solved using FEM implemented by in software developed by the authors; it also takes into accounts variable calorimetric properties. It is intent of the authors to perform several experimental tests using bi-dimensional three dimensional samples in order to increase our databases obtaining foodstuff properties and thermo physics data which would be used for further comparison. The present model will also be extended to two and three dimensional version. 

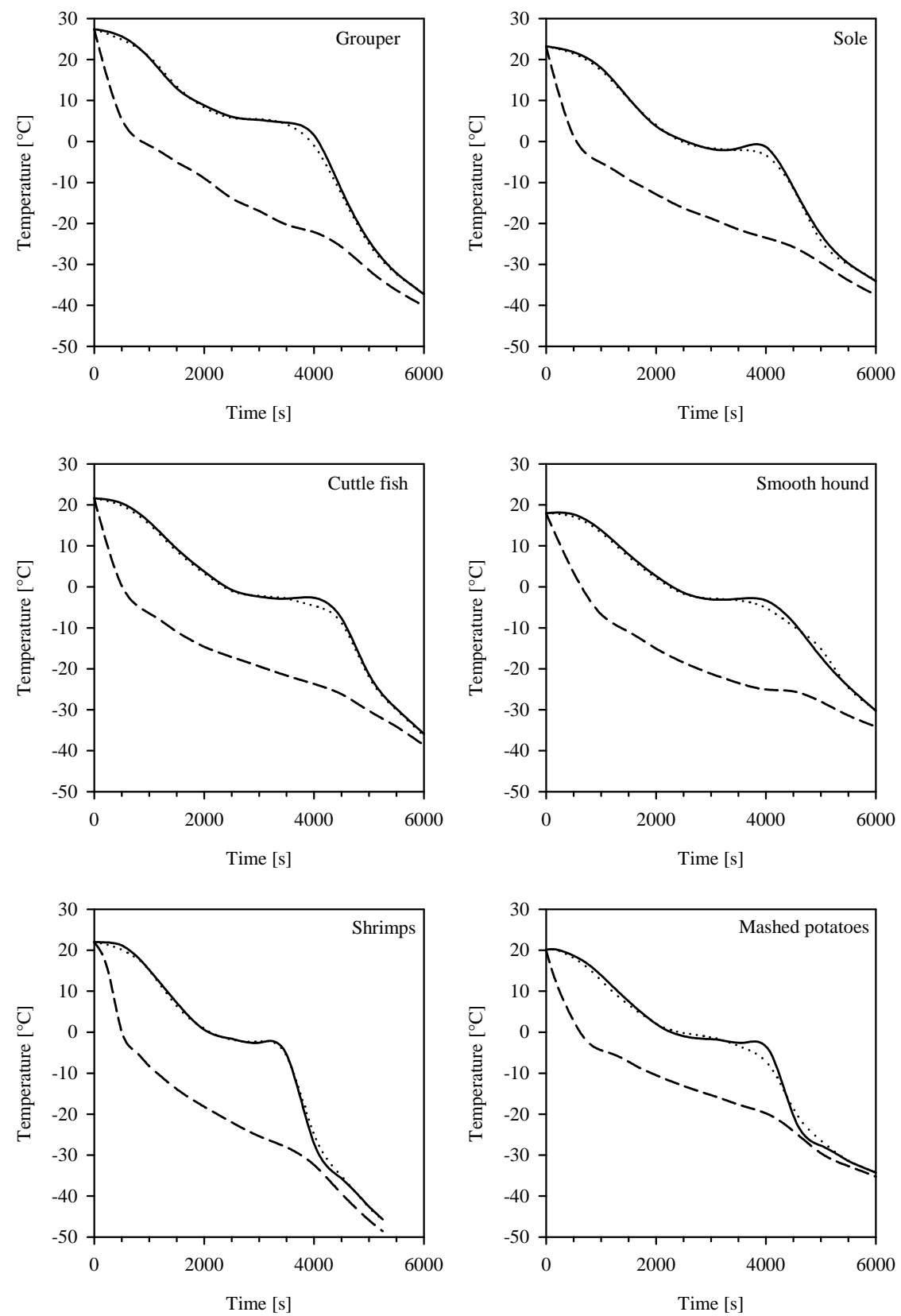

Figure 2: Temperature vs. time during freezing of several foodstuffs: surface (- - - ); centre (theoretical ——; experimental …….). 
The numerical models can be used to determine the distribution of the temperature in products undergone to advanced quick freezing processes, once the boundary conditions are fixed.

\section{References}

[1] Asdrubali, F., Cotana, F. and Messineo, A., On the evaluation of solar greenhouses efficiency in building simulation during the heating period. Energies, 5 (6), pp. 1864-1880, 2012.

[2] Messineo, A., Volpe, R. and Marvuglia, A., Ligno-cellulosic biomass exploitation for power generation: A case study in Sicily. Energy, 45, pp. 613-625, 2012.

[3] La Rocca, A., La Rocca, V., Messineo, A., Morale, M., Panno, D. and Volpe, R., Numerical solution of foodstuff freezing problems using radial basis functions. Advanced Science Letters, 19 (3), pp. 1044-1047, 2013.

[4] La Rocca, V., Panno, G., Experimental performance evaluation of a vapour compression refrigerating plant when replacing R22 with alternative refrigerants. Applied Energy, 88, pp. 2809-2815, 2011.

[5] La Rocca, V., Messineo, A., Morale, M., Dispenza, A. and Panno, D., Advanced refrigerating plants based on transcritical cycles working with carbon dioxide for commercial refrigeration. Applied Mechanics and Materials, 260-261, pp. 611-617, 2013.

[6] Messineo, A., R744-R717 cascade refrigeration system: performance evaluation compared with a HFC two-Stage system. Energy Procedia, 14, pp. 56-65, 2012.

[7] Messineo, A., Panno, D., Performance evaluation of cascade refrigeration systems using different refrigerants. International Journal of AirConditioning and Refrigeration, 20 (3), 2012.

[8] Messineo, A., La Rocca V. and Panno, G., On-site experimental study of HCFC-22 substitution with HFCs refrigerants, Energy Procedia, 14, pp. 32-38, 2012.

[9] Bonacina, C., Comini, G. and Toffan, P., Valutazione delle proprietà termofisiche delle derrate alimentari. Quaderno N. 42, Istituto di Fisica Tecnica e Laboratorio per la Tecnica del Freddo del CNR, Padova, 1973.

[10] Cleland, A.C., Food refrigeration processes, Elsevier Applied Science, London, 1990.

[11] La Rocca, V., Morale, M., The prediction of cooling and freezing of foodstuffs by the finite-element method. Proc. of the IIF/IIR Conference "Advances in the Refrigeration Systems, Food Technologies and Cold Chain”, Sofia, pp. 303-308, 1998.

[12] Rahman, M.D., Food Properties Handbook, CRC Press, London, 1995. 